\title{
La importancia de los registros de pacientes con enfermedades raras de la visión para la investigación en ciencias sociales
}

\section{The Importance of Rare Eye Disease Patient Registries for Social Science Research}

\author{
$\mathrm{M}^{\mathrm{a}}$ Teresa del Álamo Martín ${ }^{1}$ \\ Universidad de Valladolid (España) \\ ORCID: https://orcid.org/0000-0003-1273-3538
}

Recibido: 08-11-2020

Aceptado: 24-12-2020

\section{Resumen}

El propósito de este artículo ha sido evidenciar lo que las ciencias sociales pueden aportar para avanzar en el conocimiento y comprensión de los problemas personales y sociales derivados de padecer Enfermedades Raras de la visión. Se concluye que la incorporación de datos, en los Registros Nacionales de Pacientes, es una oportunidad para favorecer la investigación de los problemas existentes en nuestro contexto social y cultural, en la búsqueda de soluciones que redunde en la mejora de los tratamientos, de la atención sanitaria, psico-social, de la educación, en el empleo, etc. y en la evaluación de los resultados. En definitiva, en las condiciones y calidad de vida de los pacientes y sus familiares.

Palabras-clave: Enfermedades Raras de la visión, distrofias de retina, registro de pacientes, investigación social.

\footnotetext{
${ }^{1}$ (mariateresa.alamo@uva.es); Profesora Titular de Universidad del Departamento de Sociología y Trabajo Social de la Universidad de Valladolid. Doctora en Ciencias de la Visión. Licenciada en Derecho y Diplomada en Trabajo social. Miembro del Consejo del Instituto de Oftalmobiologia Aplicada (IOBA) de la Universidad de Valladolid y miembro de la Unidad de Investigación Social en Salud y en Enfermedades Raras. Ha publicado artículos en revistas tales como Investigative Ophthalmology \& Visual Science, Cuadernos de Trabajo social, Trabajo social y salud, etc.
} 


\begin{abstract}
The purpose of this article has been to show what social sciences can contribute to advance in the knowledge and understanding of personal and social problems derived from suffering from rare vision diseases. It concludes that the incorporation of data in the National Patient Registries is an opportunity to encourage research into the problems existing in our social and cultural context, in the search for solutions that will lead to improvements in treatment, health care, psycho-social care, education, employment, etc. and in the evaluation of results. In short, in the conditions and quality of life of patients and their families.
\end{abstract}

Key-words: Rare Diseases of Vision, Retinal Dystrophies, Patient Registration, Social Research.

\title{
Introducción
}

Disponer de información sistematizada es una necesidad generalizada en todas las disciplinas científicas. En el ámbito de las Enfermedades Raras (ER) o poco frecuentes o minoritarias o de baja prevalencia ${ }^{2}$, los registros de pacientes ${ }^{3}$ se constituyen en instrumentos clave para poder avanzar en la investigación clínica, en la mejora de la atención, en la vigilancia y en la calidad de vida de los afectados.

En el caso de las patologías raras que afectan al ojo o a la visión ${ }^{4}$ es evidente

\footnotetext{
${ }^{2}$ En Europa se consideran Enfermedades Raras las que tienen una prevalencia de 1 por 2.000 personas. En Estados Unidos, 1 por 200.000 personas.

${ }^{3}$ Un registro se define como un "sistema organizado que utiliza métodos observacionales para la recogida de datos homogéneos (normalmente clínicos, pero no exclusivamente), en una población definida para evaluar resultados específicos, con una finalidad científica, clínica o de gestión y toma de decisiones".

${ }^{4}$ Millán (2018) señala que el común denominador de todas ellas es su base genética y la inexistencia de tratamiento. Las clasifica atendiendo al tejido que se ve afectado. De modo que identifica tres tipos
} principales de patología rara ocular:

1) La patología neuro-ocular, que afecta básicamente a la retina y al nervio óptico. Dentro de esta tipología la retinosis pigmentaria es el paradigma de las distrofias de retina. Entre las patologías del nervio óptico se podrían incluir la atrofia óptica y la neuropatía hereditaria óptica de Leber. Otras distrofias de retina sindrómicas son: Síndrome de Usher en el que existe una asociación de sordera y ceguera de origen genético más prevalente; el Síndrome de Bardet-Biedl asociado a la polidactilia, obesidad, hipogenitalismo, disfunción renal y discapacidad intelectual leve; Otros síndromes extremadamente raros son los de Senior-Loken, de Joubert, o de Alström.

2) Las distrofias corneales abarcan un grupo heterogéneo de enfermedades que no tienen manifestaciones sistemáticas con clínica variable en forma de opacidades de la córnea y que afectan a la agudeza visual en diferentes grados

3) La patología que implica deficiencias en el desarrollo del globo ocular o en alguna de sus partes. Entre las patologías raras relacionada con el desarrollo ocular encontramos la anoftalmia y la microftalmia que se refiere a la ausencia completa del globo ocular o poco desarrollado 
que los registros de pacientes, en los que se incluye información detallada de la persona afectada por la enfermedad y el seguimiento de su estado de salud, son fundamentales para poder avanzar en el conocimiento de las repercusiones negativas que dichas enfermedades tienen en las personas y sus familiares para mejorar su tratamiento.

La primera dificultad que deben afrontar los investigadores que estudian la problemática de población afectada de patologías raras de la visión es la captación de la muestra para realizar estudios clínicos, debido a que muchas de ellas no obtienen un diagnóstico completo de su patología.

Un estudio realizado por Achroma (2018) una organización dedicada a la atención de la acromatopsia, concluyó que, en ocasiones, los pacientes afectados por la patología (un 58\% de los adultos y un 65\% los niños) no llegan a realizar pruebas genéticas que corroboren el diagnóstico clínico de la enfermedad. Estos pacientes buscan apoyos en las unidades de baja visión para hacer frente a las dificultades funcionales que ocasiona su patología en la realización de las actividades de la vida cotidiana. Estas prácticas, además de dificultar enormemente la búsqueda de tratamientos que puedan lograr la curación de la enfermedad, imposibilitan dimensionar la magnitud del problema al impedir conocer con exactitud la prevalencia de la patología ocular, la diversidad clínica, la distribución geográfica y la edad de las personas afectadas, la tasa de progresión de la patología en el conjunto de la población. La falta de resultados en esta línea influye negativamente en la captación de inversores que financien el desarrollo de medicamentos.

Entre los estudios que aportan evidencias sobre los beneficios de los registros de pacientes para el avance de la investigación clínica destacamos los de Mansfield, Yerxa, and Branham (2020), para el caso de las distrofias retinianas ${ }^{5}$, en las que se incluyen enfermedades huérfanas ${ }^{6}$ hereditarias de la retina como la retinosis pigmentaria, el síndrome de Usher y la enfermedad de Stargardt, y que concluyen que la implementación de los registros y de programas de acceso abierto a pruebas genéticas está siendo primordial en el avance de la investigación clínica para luchar contra la discapacidad visual y la ceguera. También, De Roach et al, 2012 concluyeron que el Registro de Pacientes con Distrofias Retinianas de Australia y el Banco de ADN son herramientas valiosas para investigar la etiología y las relaciones

respectivamente. La aniridia afecta principalmente al iris. Puede presentarse de forma aislada o asociada a otras alteraciones como el síndrome de Rieger o Axenfeld-Rieger; el síndrome de Peters, el síndrome de Gillespie, que presenta aniridia, ataxia y retraso mental, o el síndrome de WAGR, cuyo nombre corresponde a las siglas en lengua inglesa de las alteraciones presentes en el mismo (Wilms tumor, Aniridia, Genital abnormalities and Retardation).

${ }^{5}$ Las distrofias retinianas son las más numerosas, heterogéneas y complejas. Con una elevada variabilidad clínica y heterogeneidad genética.

${ }^{6}$ Las enfermedades raras también se les conoce como "enfermedades huérfanas" porque carecen de medicamentos, estudios y tratamientos.

Araucaria. Revista Iberoamericana de Filosofia, Politica, Humanidades y Relaciones Internacionales, año $23, \mathrm{n}^{\circ} 46$. Primer cuatrimestre de 2021. Pp. 391-408. ISSN 1575-6823 e-ISSN 2340-2199 https://dx.doi.org/10.12795/araucaria.2021.i46.19 
genotipo-fenotipo de las enfermedades retinianas causantes de ceguera y de morbilidad visual.

Matsunaga (2020) señala que gracias a la creación del Registro de enfermedades raras del Japón (RADDAR-J), en el que se ha incluido como categoría de enfermedad la sordoceguera, se está pudiendo recopilar y analizar los datos clínicos y genómicos para mejorar la práctica médica de estos pacientes. Una de las dificultades a las que se enfrentan las personas con sordoceguera tiene que ver con la comunicación y la interacción con los demás. Obtener un diagnóstico genético de estas personas puede proporcionar información valiosa sobre la probabilidad de progresión de su deterioro sensorial y poder preparar con tiempo suficiente modalidades de comunicación alternativas, según se produzca el deterioro sensorial. Así, por ejemplo, en el caso de pacientes con distrofias retinianas asociadas al síndrome de Usher, en los que la pérdida auditiva está presente al nacer y comienzan a mostrar pérdida de visión durante la infancia o incluso después de la edad adulta, si se considera el lenguaje de signos como único medio de comunicación, cuando tenga lugar la pérdida de visión las dificultades de comunicación van a ser extremadamente grandes. Por ello, para estos pacientes la introducción de un implante coclear, en el momento apropiado les ayudaría en la comunicación verbal auditiva después de la pérdida de visión.

Existe consenso entre la comunidad científica ${ }^{7}$ en considerar que el desarrollo de registros de pacientes, biobancos y consorcios de investigación proporcionarán muestras más grandes y generalizadas para la investigación genómica. Estas muestras sólidas procurarán evidencias para estimar la influencia genética en patologías raras relacionadas con las distrofias retinianas, defectos en el desarrollo ocular y promoverán la aplicación de diseños de estudios innovadores para desentrañar los factores de riesgo multifactoriales (ambientales o genéticos) que impiden o interrumpen el desarrollo ocular normal o que aparezcan las distrofias. Además, permitirán introducir en la investigación enfoques más innovadores para salvar y restaurar la visión, incluidas las terapias de reemplazo / aumento de genes, edición de genes, modulación de ARN, optogenética y neuroprotección basada en genes (Fillat, Río, Martínez-Santarmaría y Bueren, 2018; Shaberman y Durham, 2019).

\footnotetext{
${ }^{7}$ Fundamentalmente entre los científicos del ámbito de la salud, de la biología y la genética.
} 


\section{Repercusiones de las enfermedades raras de la visión en la vida de los pacientes y la investigación}

Existen muchos estudios que han explorado las repercusiones negativas que la pérdida de visión tiene en la vida de las personas y de sus familiares, tanto desde el punto de vista físico (Nollett et al, 2019; Stevenson, Hart, Montgomery, McCulloch y Chakravarthy, 2004), psicológico (Heesterbeek, van der Aa, van Rens, Twisk, y van Nispen, 2017; Kempen y Zijlstra, 2014; Metzelthin et al, 2017), como social (Lawrenson et al, 2018; Owsley, 2011; Smallfield et al, 2017). Cuando la pérdida de vista se debe a una enfermedad rara de la visión, a los efectos negativos de ésta en la persona que la padece, se suman factores estresantes relacionados con la etiología genética, incluidos los riesgos para otros miembros de las familias, riesgos reproductivos y la culpa y el sentimiento de culpabilidad de los padres (Sainohira et al, 2018)

Cuando se han evaluado los predictores de la ansiedad en pacientes con enfermedades raras de la visión se ha encontrado que la disminución progresiva de la función visual en adultos con retinosis pigmentaria, el estado de discapacidad legal en adultos con síndrome de Usher (Dean, Orford, Staines, McGee y Smith, 2017) y los comportamientos de ocultamiento de la identidad en adultos con retinosis pigmentaria estaban asociados con ansiedad y depresión (Anil y Garip, 2018; Zapata, 2018). Además, la prevalencia de síntomas depresivos en pacientes con retinosis pigmentaria, síndrome de Stargardt, de Usher supera a la población general (Chaumet-Riffaud et al, 2017; Moschos, Nitoda y Lavaris, 2015)

Otros estudios realizados con pacientes con retinosis pigmentaria concluyen que estos pacientes, además de afrontar los desafíos prácticos derivados de vivir con la enfermedad acompañados con estados emocionales y psicológicos de fatiga, miedo, aislamiento y vulnerabilidad (Garip y Kamal, 2019) o enfrentar sus propios juicios, también afrontan el estigma que perciben que los demás tienen sobre ellos debido a su discapacidad visual (Garip y Kamal, 2019). En el estudio de Pybis, Thurston, Dennison, Broom y Miller (2016), algunos participantes informaron haber sido tratados por otros como si tuvieran poca inteligencia, debido a su incapacidad para identificar señales sociales en la interacción con los otros, que deriva en una menor participación en los eventos sociales.

Entre los estudios que más han profundizado en la identificación de dificultades que deben afrontar las personas con síndrome de Usher destacamos el de Lønborg-Møller, Subhi y Kessel, (2020). El de Roborel de Climens et $a l$, (2020) se centra en las dificultades de estos pacientes en su actuar social. Declaran su dificultad para hablar de su enfermedad con sus amigos y sus familiares, e incluso algunos de ellos declararon que ocultaban su enfermedad. 
También reportan que sus dificultades de comunicación afectan en la interacción social con otras personas, disminuyendo significativamente.

En cuanto al desempeño de roles en la escuela, en el trabajo en las actividades de la vida diaria, las personas con síndrome de Usher tienen más dificultades para seguir el curso en los centros escolares, para realizar tareas escolares, padecen mayor absentismo escolar debido a la enfermedad, e incluso algunos de ellos refieren la presencia de actitudes negativas de sus compañeros de escuela hacia ellos llegando hasta situaciones de acoso o violencia.

En el trabajo, algunos pacientes afectados por el síndrome también manifestaron la existencia de reacciones negativas de sus colegas, así como las dificultades para la realización de determinadas tareas que repercutían en una menor productividad y una incomprensión de su situación por los empleadores.

Los jóvenes manifestaron que padecer la enfermedad ha influido en la elección de la carrera universitaria o de su profesión. Así mismo, también afectó a la necesidad de cambiar su profesión, su carrera o incluso les obligó a dejar de trabajar.

Algunas de las personas experimentan una reducción de su renta disponible debido a la necesidad de adquirir determinados dispositivos, instrumentos y/o ayudas técnicas para poder hacer frente a la realización de la vida diaria. Desde la adquisición de teléfonos móviles, dispositivos electrónicos, lupas, etc.

Lo expuesto hasta el momento evidencia las dificultades individuales y los problemas sociales que los pacientes con patología de la visión de baja prevalencia tienen en la mayoría de los países. En el caso español, el estudio de Sauces y Callado (2016) muestra las dificultades de relación con el sistema sanitario, problemas de integración social, efectos en la dinámica familiar, costes económicos, problemática educativa y alteraciones psico-emocionales, es decir los efectos sociales ocasionados por las enfermedades raras a nivel general.

Respecto de las enfermedades raras de la visión, en los últimos años están siendo las asociaciones de pacientes y familiares de afectados, las que han tomado las riendas en visibilizar la realidad de las personas que padeciendo la patología visual todavía no se ha manifestado sintomatología alguna o en aquellos que, habiéndose manifestado, la discapacidad visual se encuentre en diferentes momentos, desde una discapacidad leve y/o moderada hasta la grave en incluso la ceguera ${ }^{8}$.

${ }^{8}$ La OMS (2014) precisó aún más el término de discapacidad visual atendiendo a la función visual estableciendo cuatro categorías:

- Visión normal;

- Discapacidad visual moderada; Función visual en el mejor ojo entre una agudeza visual de entre menos de $6 / 18(0.3)$ y $6 / 60(0.1)$

- Discapacidad visual grave; se entiende una agudeza visual inferior a 6/60 (0.1) e igual o superior a 3/60 (0.05).

- Ceguera; se define como una agudeza visual de presentación inferior a 3/60 (0.05), o una pérdida del campo visual a menos de $10^{\circ}$, en el mejor ojo.

Araucaria. Revista Iberoamericana de Filosofia, Politica, Humanidades y Relaciones Internacionales, año $23, \mathrm{n}^{\circ} 46$. Primer cuatrimestre de 2021. Pp. 391-408. ISSN 1575-6823 e-ISSN 2340-2199 https://dx.doi.org/10.12795/araucaria.2021.i46.19 
En palabras de Oviedo, Hernández y Ruiz (2015) esta situación se debe a que a pesar de que existen evidencias empíricas sobre las repercusiones negativas en la vida de los pacientes y de sus familias, provocadas por la patología visual, éstas no han logrado despertar el interés de los profesionales, ni de las administraciones públicas para evidenciarla como un problema de carácter público que requiera ser intervenido. En el caso de los profesionales el estudio realizado por Álamo-Martín y Álamo-Martín (2019) evidencia el desconocimiento de los trabajadores sociales que trabajan en el ámbito privado y en el público sobre la realidad personal y socio-económica de las personas con patología visual.

Tampoco ha ayudado mucho, como señalan Toboso-Martín y RogeroGarcía, (2012) el hecho de que las investigaciones sociales sobre discapacidad hayan tratado a la discapacidad como un todo homogéneo, sin distinguir entre las diversas discapacidades, y que no se haya facilitado la participación de los actores en las mismas. Ello ha contribuido a la invisibilización de los diferentes colectivos y a que no se recoja adecuadamente información básica y necesaria sobre sus necesidades y sus circunstancias en el ámbito personal y social.

En este sentido. los investigadores de las ciencias sociales podemos contribuir a arrojar luz sobre la situación de los pacientes en temas claves dentro de los dominios del impacto psicológico, social y de la calidad de vida. A modo de ejemplo, destacamos los siguientes: formas de afrontar la enfermedad basadas en la solución de problemas o en la gestión de las emociones (entre los que se podría incluir desde las ayudas técnicas y su uso, al humor, la religión o la esperanza en el hallazgo de un futuro tratamiento); la identidad y sentido de pertenencia al grupo de afectados por patologías raras de la visión; la capacidad de vivir independientemente conforme a los deseos, aspiraciones, etc., o la capacidad jurídica para tomar decisiones en todos y cada uno de los aspectos de la vida en el presente o en el futuro'; la salud mental; las relaciones con la familia, con amigos y compañeros, con profesionales (Ulldemolins, Benach, Guisasola y Artazcoz, 2019).

En el contexto español encontramos escasas evidencias que analicen algunos de los problemas de investigación propuestos. En este sentido, los estudios con pacientes con retinosis pigmentaria proceden fundamentalmente del campo de la psicología, especialmente de la psicología evolutiva y de la

La discapacidad visual moderada y la discapacidad visual grave se reagrupan comúnmente bajo el término "baja visión", y el total de casos de discapacidad visual están representados conjuntamente por la baja visión y la ceguera (OMS, 2014).

${ }^{9}$ No olvidemos que algunas de las enfermedades raras de la visión además de la pérdida de la visión están asociadas con padecer sordoceguera y/o discapacidad intelectual y las implicaciones que esto tiene desde el punto de vista de la capacidad para prestar consentimiento y el nombramiento de tutores o curadores. Muchas de estas cuestiones se abordan en el Código civil y en la Ley de enjuiciamiento Civil. Cuestiones de la vida cotidiana que van desde el contraer matrimonio, administrar el patrimonio propio, capacidad para testar o instrucciones previas y testamento vital.

Araucaria. Revista Iberoamericana de Filosofia, Politica, Humanidades y Relaciones Internacionales, año $23, \mathrm{n}^{\circ} 46$. Primer cuatrimestre de 2021. Pp. 391-408. ISSN 1575-6823 e-ISSN 2340-2199 https://dx.doi.org/10.12795/araucaria.2021.i46.19 
educación. Dichas investigaciones se centran en el autoconcepto ${ }^{10}$ de niños y adolescentes y proporcionan algunas estrategias, de carácter psicopedagógico, para los docentes que intervienen en su educación y que su rendimiento escolar y para facilitar su inclusión social (Chacón-López y López-Justicia, 2020; López-Justicia y Chacón-López, 2015; Zapata, 2018)

Como hemos podido observar en los apartados anteriores, son muchos los problemas de investigación de carácter social para los que faltan evidencias que conduzcan a la comprensión de los mismos, y que, al día de hoy, son escasos este tipo de trabajos. También hemos aportado evidencias sobre la utilidad que los registros de pacientes están teniendo en la actualidad en países como, Estados Unidos, Australia, Dinamarca, Japón en el avance de la investigación clínica de las enfermedades raras de la visión (Brinks et al, 2019) Dichos registros han sido avalados por el Comité de Expertos en enfermedades raras Europeo (European Union Committee of Experts on Rare Diseases. EUCERD Core Recommendations on Rare Disease Patient Registration and Data Collection), al considerar que facilitar la investigación de las enfermedades raras tanto en el campo clínico como en el epidemiológico. A su vez, coadyuvan en la realización de ensayos clínicos y de estudios post-comercialización de tratamientos.

Por otro lado, dicho Comité de Expertos señala que los registros de pacientes pueden servir para informar en el desarrollo de políticas y monitorizar la atención sanitaria y de las intervenciones terapéuticas" (Zurriaga, Martínez, Corrochano y Cavero-Carbonell, 2018). Las consideraciones expuestas por dicho comité abre la entrada a la investigación a muchas de las disciplinas propias de las ciencias sociales, como la sociología, la ciencia política, el derecho, la economía o el trabajo social.

Los registros de pacientes únicos podrían contribuir a resolver alguno de los problemas que para la investigación en ciencias sociales es crucial. Nos referimos a la escasa frecuencia de las enfermedades raras de la visión, que imposibilita que una sola organización disponga de los datos suficientes para realizar investigaciones en las que sus resultados puedan ser generalizables o que no se disponga de datos sobre aquellas enfermedades llamadas ultra-raras. Nos podemos encontrar con la circunstancia de que, aun existiendo varios registros, no se dispongan de ninguna información sobre pacientes con algunas de las enfermedades existentes o que en otros podamos tener datos duplicados.

En España, el Registro de Pacientes de Enfermedades Raras 11 del Instituto de Salud Carlos III, que inicia su andadura tras la publicación de la Orden SCO/1730/2005, de 31 de mayo, estableció los criterios de su creación y funcionamiento. Desde su origen, posee naturaleza administrativa y está integrado

${ }^{10}$ El autoconcepto se puede definir como la percepción que los individuos poseen de sí mismos. Es el resultado de un proceso activo de construcción por parte del sujeto a lo largo de todo su desarrollo evolutivo. 
en el Sistema de Información Sanitaria del Sistema Nacional de Salud previsto en el artículo 53 de la Ley 16/2003, de 28 de mayo, de cohesión y calidad del Sistema Nacional de Salud. Está coordinado y dirigido desde el Instituto de Investigación en Enfermedades Raras (IIER), centro perteneciente al ISCIII y que también forma parte del CIBERER (Consorcio de Investigación Biomédica en Red de Enfermedades Raras). Desde sus inicios, este registro también está declarado en la Agencia Española de Protección de Datos, ya que contiene datos de identificación personal e información clínica sensible, que requiere el máximo nivel de seguridad.

El objetivo del registro es ofrecer a los profesionales del sistema de salud, investigadores y al colectivo de pacientes y familiares un mayor nivel de conocimiento acerca del número y distribución geográfica de las personas afectadas por enfermedades raras en España. Se persigue así fomentar la investigación y aumentar la visibilidad de estas enfermedades, y favorecer la toma de decisiones para una adecuada planificación sanitaria y una correcta distribución de recursos.

El Real Decreto 1091/2015 establece en su artículo 3 que el Registro tiene por finalidad:

a) Proporcionar información epidemiológica sobre las enfermedades raras, sobre la incidencia y prevalencia de las mismas y sobre sus factores determinantes asociados.

b) Facilitar la información necesaria para orientar la planificación y gestión sanitaria y la evaluación de las actividades preventivas y asistenciales en el ámbito de las enfermedades raras.

c) Proveer los indicadores básicos sobre enfermedades raras que permitan la comparación entre las comunidades autónomas y con otros países.

A fecha de octubre de 2020, el registro cuenta con 20.957 pacientes con enfermedades raras. La Tabla I muestra el número de pacientes registrados con enfermedades raras de la visión.

\begin{tabular}{|l|l|}
\hline Tabla $1 . \mathrm{N}^{\mathrm{o}}$ de pacientes registrados por Enfermedad Rara de la visión \\
\hline Enfermedad Rara de la Visión & $\mathrm{N}^{\mathrm{o}}$ de Pacientes Registrados \\
\hline Retinitis pigmentosa & 98 \\
\hline Amaurosis congénita de Leber & 14 \\
\hline Aniridia & 24 \\
\hline Enfermedad de Stargard & 29 \\
\hline Neuropatía óptica hereditaria de Leber & 12 \\
\hline Síndrome de Usher & 12 \\
\hline Síndrome de Usher tipo 1 & 1 \\
\hline Síndrome de Usher tipo 2 & 3 \\
\hline Síndrome WAGR & 2 \\
\hline
\end{tabular}


Elaboración propia a partir de los datos obtenidos del Registro de Pacientes de Enfermedades Raras Instituto Carlos III Octubre de 2020

La incorporación de información se hace mediante tres vías: 1) a partir de la introducción directa del paciente por parte del propio afectado o de un familiar o representante; 2) gracias a los profesionales que incluyen la información de sus bases de datos (ya sean investigadores o sociedades médicas) y 3 ) a través de los sistemas de información sanitaria de las administraciones

La entrada de casos por parte de las dos primeras vías, es decir, ciudadanos o profesionales, responde a lo que habitualmente se define como registro de pacientes. Aunque, este tipo de registros, no contienen la información de todas las personas que padecen una enfermedad concreta, sí son muy útiles para la investigación, fundamentalmente por su nivel de detalle y por las posibilidades que ofrece. Entre otras razones, permite el seguimiento más completo al disponer de más información sobre la evolución de los pacientes y las características de la enfermedad, (factores, pronóstico, supervivencia, uso de medicamentos, calidad de vida, análisis de la dependencia, uso de recursos sanitarios, entre otras). Así mismo, permite el reclutamiento para realizar estudios clínicos específicos, ya que se cuenta con el consentimiento informado de todas las personas afectadas por enfermedades raras incluidas en el registro permitiendo así contactar con aquellas que estén interesadas en participar en ensayos clínicos, donación de muestras biológicas, etc.

Pues bien, tras lo indicado antes conviene señalar algo más. Si se pretende identificar los factores determinantes sociales de la salud, el acceso al registro puede ser una oportunidad para obtener dicha información. A su vez, permite tener la posibilidad de contactar directamente con los pacientes; lo que podría proporcionar información valiosa en torno a la percepción que de ellos poseen de la enfermedad a lo largo de su vida. La percepción subjetiva de los acontecimientos de nuestra vida no solo están regidos por nuestras vivencias, sino también por las políticas y por los sistemas económicos, etc. Las condiciones anteriores pueden ser altamente inequitativas y dar lugar a diferencias en materia de salud. Es posible que sea inevitable que algunas de dichas condiciones sean diferentes, en cuyo caso se consideran desigualdades, o que, de hecho, estas diferencias puedan ser innecesarias y evitables, en cuyo caso se consideran inequidades $\mathrm{y}$, por consiguiente, metas apropiadas para políticas destinadas a aumentar la equidad (OMS, 2005).

De cualquier manera, y desde una perspectiva puramente metodológica, conviene tener presente que la información obtenida no pueda ser considerada representativa de la población afectada por la Enfermedad Rara de la Visión para investigaciones de carácter cuantitativo. Ahora bien, sí podría saturar la muestra en términos de investigación cualitativa. Por otro lado, incluso permitiría triangular investigaciones mixtas en las que los datos cualitativos 
obtenidos de estos registros, pudieran completar otros datos obtenidos de Registros de Enfermedades Raras o de base poblacional que analizaremos a continuación. Por todo ello, este tipo de artefactos (los registros) abren las puertas a un conocimiento más exhaustivo y completo de las enfermedades raras visuales o no. Incluso permitiría hacer una historia del presente con mayor facilidad. Ahora bien, todo ello implicaría la necesidad de abrir estos registros a ámbitos disciplinarios propios de las ciencias sociales y humanas.

La solicitud la puede hacer cualquier ciudadano, esté afectado por la enfermedad u otra que esté autorizada para hacerlo en su nombre (familiar o representante). En la página web, https://registroraras.isciii.es/ aparecen los pasos a seguir y se accede al formulario de solicitud. Una vez que está rellenado, se acompaña de la firma del consentimiento informado y de una copia del informe médico que confirme el diagnóstico de la Enfermedad Rara que refiere. Una vez que desde el IIER se comprueba que la solicitud cumple los requisitos y que es efectivamente una Enfermedad Rara, se procede a incluir el nuevo caso en el registro, y se asigna a la persona interesada un usuario y contraseña de acceso a la plataforma online. El registro proporciona a los usuarios información específica asociada a la Enfermedad Rara por la que se han dado de alta, manteniendo así la filosofía del registro como un sistema de retroalimentación de la información a los pacientes y sus familiares.

Para los investigadores y profesionales sanitarios el registro se convierte en una oportunidad para gestionar la información relativa a una o varias enfermedades raras. Esta gestión se realiza en colaboración con los administradores del sistema en el ISCIII-IIER y cuenta con las debidas garantías de confidencialidad y seguridad ${ }^{11}$.

La tercera vía de entrada de datos, se produce a través de los sistemas de información sanitaria existentes. Estos son los que se denominan registros de base poblacional, que a diferencia de los de pacientes, sí recogen la información total de personas afectadas por una enfermedad rara concreta aunque basándose en información sanitaria. Esta tercera alternativa posee más registros que los de pacientes y se dirigen a la vigilancia de la salud, proporcionando información sobre la evolución de las enfermedades. En esta vía colabora la Red Española de Registros de Enfermedades Raras para la Investigación (SpainRDR, por sus siglas en inglés).

Los registros de base poblacional pueden estar afectados por determinados sesgos de selección relacionados con el método de elección de los centros donde se realiza la obtención de la información, así como con la selección de especialistas interesados en la investigación clínica de las enfermedades raras de la Visión. Lo dicho es importante, puesto que es posible que los enfermos de

\footnotetext{
${ }^{11}$ Información extraída de la página web del Registro de Pacientes de Enfermedades Raras Instituto Carlos III. Octubre de 2020. https://registroraras.isciii.es/
}

Araucaria. Revista Iberoamericana de Filosofí, Política, Humanidades y Relaciones Internacionales, año $23, \mathrm{n}^{\circ} 46$. Primer cuatrimestre de 2021. Pp. 391-408. ISSN 1575-6823 e-ISSN 2340-2199 https://dx.doi.org/10.12795/araucaria.2021.i46.19 
los centros sanitarios sean los más graves o estén mejor estudiados que aquellos registrados en otros lugares. También pueden existir diferencias en la derivación a las consultas de oftalmología en función de distintos factores como, por ejemplo, el nivel asistencial o la presencia de determinadas manifestaciones órgano-específicas, que motiven la derivación a otras especialidades. Los registros de base poblacional sí incluyen elevados números de centros participantes con amplia distribución geográfica y diversas características de los centros participantes, es posible que minimicen los sesgos de selección. Por ello, es importante la diversificación del origen de los datos, en los que se incluyan pacientes procedentes del ámbito hospitalario, pero también de la atención primaria o de la sociosanitaria.

\section{Aspectos éticos, legales a considerar en la investigación social con personas con enfermedades raras de la visión}

Para terminar, es necesario abordar algunas cuestiones de carácter legal y ético que están presentes en la investigación con pacientes de enfermedades raras de la vista.

En primer lugar, señalar, que la Ley de Investigación Biomédica 14/2007, la Ley Orgánica de Protección de Datos, LOPD, 15/1999, y el Real Decreto $1716 / 2011$ resaltan el hecho de que los intereses de los seres humanos prevalecen sobre los intereses de la sociedad y de la ciencia.

Los riesgos derivados de la participación como sujetos de investigación y de la incorporación en Registros de Pacientes de Enfermedades Raras, pueden deberse a la violación de la confidencialidad y de la privacidad de estas personas que, desde todos los puntos de vista, pueden ser considerados vulnerables. Dicha caracterización proviene de lo que Zurriaga et al, (2018) denomina como riesgos intrínsecos. A estos, habría que añadir los llamados riesgos ulteriores, debido a que en los registros de pacientes se puedan generar una cantidad importante de datos en el fututo que deban someterse a una elevada protección de la confidencialidad para evitar el potencial riesgo de discriminación genética.

Por otro lado, y dentro de estos riesgos ulteriores, también debemos ser conscientes que la población con enfermedades raras visuales son particularmente vulnerables y requieren protección especial. Decimos esto, puesto que algunas de estas personas no están en disposición de otorgar o rechazar el consentimiento por sí mismos Decimos esto, puesto que algunas de estas personas no están en disposición de coerción o influencia indebida (Zurriaga et al, 2018). Toda esta situación necesita de ulteriores análisis de carácter ético para poder dilucidar las decisiones futuras en este sentido. 
En el campo de la investigación en ciencias sociales, el concepto de vulnerabilidad es una noción escasamente abordada tanto en la bibliografía especializada, como en las guías éticas. Si bien se realizan numerosas investigaciones con grupos sumamente vulnerables, el análisis de dicho concepto y su vinculación con la investigación social no ha sido problematizado suficientemente. En este ámbito no existe una guía o código de ética que sea aplicado y exigido internacionalmente, sino que en cada país varía el estatus de protección de los aspectos éticos de estas investigaciones y el enfoque de las distintas cuestiones éticas. Algunas guías de las ciencias sociales se refieren a individuos y grupos vulnerables. Generalmente, se asocia la vulnerabilidad a la disminución de las capacidades cognitivas (Social Research Association, 2003) o a la edad del participante. Por ejemplo, el Código de ética de la Asociación Americana de Sociología (ASA, por sus siglas en inglés) afirma lo siguiente: "Al llevar a cabo una investigación con poblaciones vulnerables (por ejemplo, con jóvenes, con poblaciones que han migrado recientemente, con enfermos mentales), los sociólogos deben tener especial cuidado para asegurar que se comprenda la naturaleza voluntaria de la participación en la investigación y que el consentimiento obtenido no sea coaccionado" (American Sociological Association, 2018).

La Ley de investigación Biomédica obliga a que toda muestra debe ir acompañada de un documento que contenga el consentimiento informado, que la ley define como "un acuerdo por escrito mediante el cual el sujeto de investigación o, en su caso, su representante legal autoriza su participación en la investigación con pleno conocimiento de la naturaleza de los procedimientos, beneficios y riesgos a que se someterá, con la capacidad de libre elección y sin coacción alguna".

También para poder contactar y hacerles propuestas para que participen en las investigaciones se exige que éstos presten su consentimiento.

Aparte de la vulnerabilidad y la exigencia de no haber obtenido el consentimiento con violencia, la necesidad de proteger la identidad del paciente es otra de las cuestiones que deben abordarse tanto desde la ética como desde el ámbito jurídico a fin de preservar el derecho a la intimidad de los pacientes. Las dificultades mayores se presentan especialmente en los casos de enfermedades ultra-raras. Otra de las cuestiones éticas y jurídicas ligadas a la protección de los datos del paciente deriva de la necesidad de implementar un identificador único de paciente, especialmente en lo que se refiere a su utilización y a la protección de datos (Thompson et al, 2014).

Un debate ético más es la disponibilidad de las organizaciones e investigadores participantes a compartir, por un lado, los datos y por otro los resultados de las investigaciones. 
También desde las organizaciones de pacientes se está debatiendo la necesidad de que se impliquen los pacientes en la gobernanza y gestión de los registros, en el papel que podrían jugar los registros para la construcción y el empoderamiento de pacientes, o en la colaboración de lo público y lo privado para asegurar la sostenibilidad de los registros.

\section{Conclusión}

Los registros de pacientes dentro de las políticas desarrolladas en los últimos años en favor de conocer y visibilizar las enfermedades raras de la visión han sido una prioridad global, aunque de momento sólo se haya iniciado el camino y es evidente que queda mucho por hacer.

Se ha establecido claramente el propósito y la justificación de los registros, siendo el papel de las ciencias sociales clave al colaborar con las ciencias de la salud en la identificación de los determinantes sociales de la salud y para proporcionar la información necesaria a la hora de planificar, gestionar y evaluar las acciones que se pongan en marcha a fin de la prevención y asistencia de las enfermedades raras de la visión. Tomando al paciente como el centro de todas las actuaciones que se lleven a cabo.

Aunque, desde la aparición de los registros de pacientes en los últimos años, estos han ido creciendo y desarrollándose, todavía quedan aspectos legales y éticos de los registros que deben profundizarse y resolverse.

Hemos visto las dificultades y los retos que las ciencias sociales deben afrontar en el campo de las enfermedades raras de la visión, pero no dejan de ser oportunidades que deben aprovecharse para avanzar en el conocimiento y tratamiento de las mismas en interés de los pacientes y sus familiares. 


\section{Referencias bibliográficas:}

Achroma Corp. (2018). Achroma Corp. announces global survey results of 226 people with achromatopsia.

Álamo-Martín, M. ${ }^{\text {a }}$ T. del y Álamo-Martín, M.A. del. (2019). Visibilizando a las personas con patología ocular grave para el Trabajo Social Sanitario y la defensa de sus derechos. Trabajo Social y Salud, 92, 5-20.

American Sociological Association. (2018). Code of Ethics Code of Ethics. Code of Ethics, 104(911), 1-21. Retrieved from https://nhaa.org.au/about/ code-of-ethics-constitution?highlight=WyJjb2Rlliwib2YiLCJldGhpY3M iLCJjb2R1IG9mIiwiY29kZSBvZiBldGhpY3MiLCJvZiBldGhpY3MiX $\mathrm{Q}==\% 0$ Ahttp://www.aamft.org/imis15/Content/Legal_Ethics/Code_of_ Ethics.aspx

Anil, K. y Garip, G. (2018). Coping strategies, vision-related quality of life, and emotional health in managing retinitis pigmentosa: A survey study. $B M C$ Ophthalmology, 18(1), 1-12. http://doi.org/10.1186/s12886-018-0689-2

Brinks, M.V., Redd, T., Lambert, W.E., Zaback, T., Randall, J., Field, T. y Wilson, D. (2019). Using registry data to characterize the incidence and causes of blindness in Oregon. PLoS ONE, 14(8), 1-9. http://doi. org/10.1371/journal.pone.0220983

Chacón-López, H. y López-Justicia, M.D. (2020). Self-Concept difficulties according to sex and studies of young people with retinitis pigmentosa. Revista Española de Orientacion y Psicopedagogia, 31(1), 98-112. http:// doi.org/10.5944/reop.vol.31.num.1.2020.27292

Chaumet-Riffaud, A.E., Chaumet-Riffaud, P., Cariou, A., Devisme, C., Audo, I., Sahel, J.A., y Mohand-Said, S. (2017). Impact of Retinitis Pigmentosa on Quality of Life, Mental Health, and Employment Among Young Adults. American Journal of Ophthalmology, 177, 169-174. http://doi. org/10.1016/j.ajo.2017.02.016

Dean, G., Orford, A., Staines, R., McGee, A., y Smith, K.J. (2017). Psychosocial well-being and healthrelated quality of life in a UK population with Usher syndrome. BMJ Open, 7(1), 1-9. http://doi.org/10.1136/ bmjopen-2016-013261

De Roach, J., McLaren, T., Paterson, R., O’Brien, E., Hoffman, L., Mackey, D.A., ... Lamey, I. (2012). Establishment and evolution of the Australian Inherited Retinal Disease Register and DNA Bank. Clinical and Experimental Ophthalmology, 41(5), 476-483.

Fillat, C., Río, M. del, Martínez-Santarmaría, L. y Bueren, J.A. (2018). Terapias avanzadas en Enfermedades Raras. Arbor, 194(789), a467. 
Garip, G. y Kamal, A. (2019). Systematic review and meta-synthesis of coping with retinitis pigmentosa: Implications for improving quality of life. $B M C$ Ophthalmology, 19(1), 1-16. http://doi.org/10.1186/s12886-019-1169-z

Heesterbeek, T. J., van der Aa, H. P. A., van Rens, G.H.M.B., Twisk, J.W.R. y van Nispen, R.M.A. (2017). The incidence and predictors of depressive and anxiety symptoms in older adults with vision impairment: a longitudinal prospective cohort study. Ophthalmic and Physiological Optics. http://doi. org/10.1111/opo.12388

Kempen, G.I.J.M. y Zijlstra, G.A.R. (2014). Clinically relevant symptoms of anxiety and depression in low-vision community-living older adults. American Journal of Geriatric Psychiatry, 22(3), 309-313. http://doi. org/10.1016/j.jagp.2012.08.007

Lawrenson, J.G., Graham-Rowe, E., Lorencatto, F., Burr, J., Bunce, C., Francis, J. J., ... Grimshaw, J.M. (2018). Interventions to increase attendance for diabetic retinopathy screening. Cochrane Database of Systematic Reviews. http://doi.org/10.1002/14651858.CD012054.pub2

Lønborg-Møller, H., Subhi, Y. y Kessel, L. (2020). Living with Usher Syndrome: Patient and Physician Perspectives. Ophthalmology and Therapy, 9(3). http://doi.org/10.1007/s40123-020-00258-6

López-Justicia, M.D., y Chacón-López, H. (2015). Cambios emocionales asociados a la edad y género en personas con retinosis pigmentaria. Revista Argentina de Clinica Psicologica, XXIV(August), 57-66.

Mansfield, B.C., Yerxa, B.R., y Branham, K.H. (2020). Implementation of a registry and open access genetic testing program for inherited retinal diseases within a non-profit foundation. American Journal of Medical Genetics, Part C: Seminars in Medical Genetics, (June), 838-845. http:// doi.org/10.1002/ajmg.c.31825

Matsunaga, T. (2020). Clinical genetics, practice, and research of deafblindness: From uncollected experiences to the national registry in Japan. Auris Nasus Larynx, (xxxx). http://doi.org/10.1016/j.anl.2020.08.017

Metzelthin, S.F., Zijlstra, G.A., van Rossum, E., de Man-van Ginkel, J.M., Resnick, B., Lewin, G., ... Kempen, G.I. (2017). "Doing with ...” rather than "doing for ..." older adults: rationale and content of the "Stay Active at Home" programme. Clinical Rehabilitation, 26921551769873. http:// doi.org/10.1177/0269215517698733

Millán Salvador, J.M. (2018). Enfermedades Raras de la visión. Arbor, 194(789), 462. http://doi.org/10.3989/arbor.2018.789n3004

Moschos, M.M., Nitoda, E. y Lavaris, A. (2015). Estimation of depression prevalence in patients with stargardt disease using PHQ-9 and zung scores. European Journal of Ophthalmology, 26(3), 268-272. http://doi. org/10.5301/ejo.5000700 
Nollett, C., Ryan, B., Bray, N., Bunce, C., Casten, R., Edwards, R.T., ... Margrain, T.H. (2019). Depressive symptoms in people with vision impairment: a cross-sectional study to identify who is most at risk. $B M J$ Open, 9(1), e026163. http://doi.org/10.1136/bmjopen-2018-026163

OMS. (2005). Determinantes sociales de la salud. Retrieved from http://www. who.int/social_determinants/es/.

Oviedo, M. del P., Hernández, M.L. y Ruíz, M. (2015). Baja visión en Colombia: una situación invisible para el país. Revista Facultad Nacional de Salud Pública., 33(1), 22-30. Retrieved from http://aprendeenlinea.udea.edu.co/ revistas/index.php/fnsp/article/view/16499/20779829

Owsley, C. (2011). Aging and vision. Vision Research. http://doi.org/10.1016/j. visres.2010.10.020

Pybis, J., Thurston, M., Dennison, C.M., Broom, M. y Miller, A. (2016). The nature of emotional support and counselling provision for people with sight loss in the United Kingdom. British Journal of Visual Impairment, 34(2), 169-178. http://doi.org/10.1177/0264619616633884

Roborel de Climens, A., Tugaut, B., Piscopo, A., Arnould, B., Buggage, R. y Brun-Strang, C. (2020). Living with type I Usher syndrome: insights from patients and their parents. Ophthalmic Genetics, 41(3), 240-251. http:// doi.org/10.1080/13816810.2020.1737947

Sainohira, M., Yamashita, T., Terasaki, H., Sonoda, S., Miyata, K., Murakami, Y., ... Sakamoto, T. (2018). Quantitative analyses of factors related to anxiety and depression in patients with retinitis pigmentosa. PLoS ONE, 13(4), 1-9. http://doi.org/10.1371/journal.pone.0195983

Sauces, M.O.S. y Callado, R.R. (2016). Las Enfermedades Raras en España. Un enfoque social. Prisma Social, (17), 373-395.

Shaberman, B. y Durham, T. (2019). The foundation fighting blindness plays an essential and expansive role in driving genetic research for inherited retinal diseases. Genes, 10(7). http://doi.org/10.3390/genes10070511

Smallfield, S., Berger, S., Hillman, B., Saltzgaber, P., Giger, J. y Kaldenberg, J. (2017). Living with low vision: Strategies supporting daily activity. Occupational Therapy in Health Care, 31(4), 312-328. http://doi.org/10.1 080/07380577.2017.1384969

Stevenson, M.R., Hart, P.M., Montgomery, A.M., McCulloch, D.W. y Chakravarthy, U. (2004). Reduced vision in older adults with age related macular degeneration interferes with ability to care for self and impairs role as carer. British Journal of Ophthalmology, 88.

Toboso-Martín, M., y Rogero-García, J. (2012). "Diseño para todos" en la investigación social sobre personas con discapacidad. Revista Española de Investigaciones Sociológicas, 163-172. http://doi.org/10.5477/cis/ reis. 140.163 
Ulldemolins, A.R., Benach, J., Guisasola, L. y Artazcoz, L. (2019). Why are there gender inequalities in visual impairment? European Journal of Public Health, 29(4), 661-666. http://doi.org/10.1093/eurpub/cky245

Zapata, M. (2018). Personal Disability Identity in Retinitis Pigmentosa. Rehabilitation Psychology, 63(4). http://doi.org/10.1037/rep0000238

Zurriaga, Ó., Martínez, J., Corrochano, V. y Cavero-Carbonell, C. (2018). Registros y biobancos de Enfermedades Raras. Una oportunidad para avanzar.Arbor, 194(789),469.http://doi.org/10.3989/arbor.2018.789n3011 